\title{
BiPAD: Binomial Point Process Based Energy-Aware Data Dissemination in Opportunistic D2D Networks
}

\author{
Seho Han ${ }^{1}$, Kisong Lee ${ }^{2}$, Hyun-Ho Choi ${ }^{3}$ (1) and Howon Lee ${ }^{3,4, *}$ (1) \\ 1 Department of Computer Science, Yonsei University, Seoul 03722, Korea; dbgmltjd000@gmail.com \\ 2 School of Information and Communication Engineering, Chungbuk National University, \\ Chungbuk 28644, Korea; kslee851105@gmail.com \\ 3 Department of Electrical, Electronic and Control Engineering, Hankyong National University, \\ Gyeonggi 17579, Korea; hhchoi@hknu.ac.kr \\ 4 Qualcomm Institute, University of California, San Diego, CA 92093-0021, USA \\ * Correspondence: hwlee@hknu.ac.kr or hwlee@eng.ucsd.edu; Tel.: +82-31-670-5198
}

Received: 1 July 2018; Accepted: 6 August 2018; Published: 9 August 2018

\begin{abstract}
In opportunistic device-to-device (D2D) networks, the epidemic routing protocol can be used to optimize the message delivery ratio. However, it has the disadvantage that it causes excessive coverage overlaps and wastes energy in message transmissions because devices are more likely to receive duplicates from neighbors. We therefore propose an efficient data dissemination algorithm that can reduce undesired transmission overlap with little performance degradation in the message delivery ratio. The proposed algorithm allows devices further away than the $k$-th furthest distance from the source device to forward a message to their neighbors. These relay devices are determined by analysis based on a binomial point process (BPP). Using a set of intensive simulations, we present the resulting network performances with respect to the total number of received messages, the forwarding efficiency and the actual number of relays. In particular, we find the optimal number of relays to achieve almost the same message delivery ratio as the epidemic routing protocol for a given network deployment. Furthermore, the proposed algorithm can achieve almost the same message delivery ratio as the epidemic routing protocol while improving the forwarding efficiency by over $103 \%$ when $k \geq 10$.
\end{abstract}

Keywords: device-to-device (D2D) communication; data dissemination; relay selection; $k$-th furthest distance; binomial point process (BPP)

\section{Introduction}

According to the Working Party 5D (WP5D) of the International Telecommunication Union Radiocommunication Sector (ITU-R), a total of eight parameters are considered to be key to the capabilities of fifth generation mobile communications (5G): (1) peak data rate, (2) user experienced data rate, (3) latency, (4) mobility, (5) connection density, (6) energy efficiency, (7) spectrum efficiency and (8) area traffic capacity [1]. In order to satisfy these requirements, several enabling technologies for $5 \mathrm{G}$ have been proposed and actively investigated, including ultra-dense networks (UDNs), device-to-device communications (D2D), full duplex (FD), mmWave communications, massive MIMO, and so on [2].

One of the main challenges in $5 \mathrm{G}$ is the support of reliable and seamless communication between users. In particular, opportunistic D2D communications have been proposed as one of the $5 \mathrm{G}$ candidate technologies for supporting reliable 5G communications [3-8]. D2D communication technology could provide high data rates with lower energy consumption due to the relatively short transmission ranges involved, while it may increase the latency due to the multihop transmission among end devices without a base station (BS). It could also mitigate traffic bottlenecks at the BS through traffic offloading. 
It even has a use in disaster scenarios, where D2D communications can be operated without the need for BSs [9-13]. In addition, with respect to energy-efficient end-to-end (E2E) message delivery, routing and service frameworks in wireless communications considering cloud-based Internet of Things (IoT) services, many related research issues are actively investigated [14-18].

Opportunistic D2D transmission algorithms are generally classified either as flooding-based (e.g., epidemic routing [19]) or greedy-based algorithms (e.g., delegation forwarding [20], PROPHET [21] and BUBBLE-Rap [22]). In the flooding-based algorithm, all devices broadcast received messages to their neighboring devices regardless of whether or not the neighboring devices have already received them. In a greedy-based algorithm, only some devices are allowed to forward received messages, and such relay devices are determined by greedy-based relay selection criteria. The epidemic routing protocol is a simple flooding algorithm for ad-hoc networks [19] and was proposed to improve network connectivity in all settings, including desert, sea and disaster scenarios. Under this protocol, the source generates and sends a message to neighboring devices within its transmission range; the receivers then also forward the received message to their neighboring devices within their own transmission range. More specifically, the transmitter sends its summary vector, which indicates the presence or absence of the message. Through the received summary vector, receivers can check their own status and request missing or unreceived messages. Neighboring devices can then send the requested message to receivers. By repeating this procedure several times, messages can be rapidly distributed to all devices in an entire network. Although the epidemic routing protocol can maximize the message delivery ratio, defined as the ratio of the number of devices that receive messages to the total number of devices, it still causes excessive overlaps of transmission ranges among devices.

In this paper, we aim to show how the problem of excessive coverage overlap can be overcome, while nevertheless ensuring a message delivery ratio as high as can be achieved by the epidemic routing protocol. On the basis of the results in [23] (note that the probability density function (pdf) of the distance between random points and the $k$-th furthest point was analyzed in [23], where points are uniformly and randomly distributed), we propose a binomial point process (BPP)-based energy-aware data dissemination algorithm (BiPAD) in an opportunistic D2D network, to mitigate against transmission coverage overlaps, as well as to enhance the message delivery ratio. The specific advantage of our scheme is that those devices further away than the $k$-th furthest distance from the source device must participate in the delivery of the message to their neighbors.

The remainder of this paper is organized as follows. In Section 2, we present the $n$-th nearest distance from the source device using BPP and propose some of the detail of the procedure to be followed by the BiPAD algorithm, namely (1) estimation of the total number of devices, (2) calculation of the distance from the transmitter to the furthest $k$-th device and (3) making the distributed forwarding decision. In Section 3, we describe the performances of the proposed algorithm with respect to the message delivery ratio, the total number of received messages and the forwarding efficiency compared to the conventional epidemic routing protocol. In Section 4, we present some conclusions.

\section{BiPAD: Binomial Point Process-Based Energy-Aware Data Dissemination}

\section{1. $n$-th Nearest Distance by BPP}

By analyzing the BPP, we can calculate the distance from an arbitrary source device to the $k$-th furthest point. If $R_{n}$ is the distance between the arbitrary source device and the $n$-th nearest device, $f_{R_{n}}(r)$ is the probability density function (pdf) of $R_{n}$, and $F_{R_{n}}(r)$ is the cumulative distribution function (cdf) of $R_{n}$. Then, $F_{R_{n}}(r)$ can be interpreted as the probability that the number of points within $R_{n}$ is less than $n$ [23], which can be represented as:

$$
F_{R_{n}}(r)=\sum_{i=0}^{n-1}\left(\begin{array}{c}
N \\
i
\end{array}\right) p^{i}(1-p)^{N-i}, 0 \leq r \leq R,
$$


where $N$ is the total number of points in the entire network, $R$ is the radius of the overall coverage and $p$ is the probability that a point is within the radius $r$.

According to [24], the binomial random variable can be converted into an incomplete beta function as follows.

$$
F_{R_{n}}(r)=I_{1-p}(N-n+1, n)=\frac{\int_{0}^{1-p} t^{N-n}(1-t)^{n-1} d t}{B(N-n+1, n)}
$$

where the beta function, $B(a, b)$, is given by $B(a, b)=\frac{\Gamma(a) \Gamma(b)}{\Gamma(a+b)}$ and $\Gamma(\cdot)$ indicates the gamma function. Using (2), $f_{R_{n}}(r)$ can be calculated as:

$$
f_{R_{n}}(r)=-\frac{\partial F_{R_{n}}(r)}{\partial r}=\frac{d p}{d r} \frac{(1-p)^{N-n} p^{n-1}}{B(N-n+1, n)} .
$$

From (3), the expected value of $R_{n}\left(E\left[R_{n}\right]\right)$ can be represented by:

$$
\begin{aligned}
E\left[R_{n}\right] & =\frac{2}{R} \frac{1}{B(N-n+1, n)} \int_{0}^{R}\left[r\left(\frac{r}{R}\right)^{2 n-1}\left(1-\left(\frac{r}{R}\right)^{2}\right)^{N-n}\right] d r \\
& = \begin{cases}\frac{R \cdot n^{\frac{1}{2}}}{(N+1)^{\frac{1}{2}}}, & \text { if } n+\frac{1}{2}>0 \\
\infty, & \text { otherwise }\end{cases}
\end{aligned}
$$

where $t=\left(\frac{r}{R}\right)^{2}, B_{0}(a, b)=\left\{\begin{array}{ll}0, & \operatorname{Re}(a)>0 \\ -\infty, & \operatorname{Re}(a) \leq 0\end{array}\right.$ and $B_{1}(a, b)=B(a, b)$ if $\operatorname{Re}(b)>0$.

Moreover, from $n=N-k+1$, the $k$-th furthest distance from the source device can be calculated from the $\mathrm{n}$-th nearest distance.

\subsection{Distributed Relay Selection and BPP-Based D2D Data Dissemination}

We herein propose the furthest- $k$ relay selection algorithm from the $n$-th nearest distance in (4). From (4), the distance from the source to the $k$-th furthest device can be described as:

$$
r_{k}=\frac{\bar{R} n^{1 / 2}}{(\bar{N}+1)^{1 / 2}}
$$

where $k=\bar{N}-n+1$ and $\bar{N}=N \times \frac{\pi \bar{R}^{2}}{\pi R^{2}}$. Furthermore, $\bar{R}$ and $\bar{N}$ indicate the transmission range of the transmitter and the number of receivers within its transmission range, respectively. The overall procedures of the proposed algorithm can be described as follows.

\section{Start of data dissemination}

Step 1. A source device sends a message, including its location information and the desired number of relays, $k$. Here, $k$ is determined as the number required to achieve the same message delivery ratio as the epidemic routing algorithm.

\section{Calculation of $r_{k}$}

Step 2. If a device receives the message from the source, it calculates the $k$-th furthest distance from the source device to the furthest $k$-th device $\left(r_{k}\right)$ using the received $k$ value and the total number of devices within the transmission range of the receiver, as estimated by the network user density.

\section{Distributed forwarding decision and termination}

Step 3. The receiver calculates the distance from the source device to itself, $r_{\text {user }}$. If $r_{\text {user }} \geq r_{k}$, the receiver prepares to forward the received message including its location information 
and the $k$ value to its neighboring devices. Then, the receiver forwards this message to its neighbors. In our proposed BiPAD algorithm, using the $r_{k}$ value, the source device can select almost exactly the number of relay devices using this distributed forwarding decision based on BPP.

Step 4. Repeat Step 1 to Step 3 until the messages are disseminated to the entire network.

To calculate (5) in the BPP, the source device must know the exact or approximate number of neighbors within its transmission range. To this end, the source device can use the average device density of the network as a whole to estimate the number of neighbor devices within the range.

Figure 1 shows an example of the furthest-krelay selection of the proposed BiPAD algorithm when $\bar{N}=5$ and $k=4$. One example of a D2D data dissemination scenario is that of a D2D-based rescue request. In our proposed algorithm, the source device generates and transmits a message that includes the location information of the source device (i.e., global positioning system (GPS) value) and the $k$ value to all the devices within its transmission range. Provided that the neighboring devices receive the message from the source device, the devices can calculate the $k$-th furthest distance from the source device. Alternatively, instead of the $k$ value, the source device can directly transmit the $k$-th furthest distance from the source device. Each device can check its location through GPS. Devices that receive the message from the source device then know whether to forward the message or not. In other words, devices located beyond the $k$-th furthest distance forward the message to neighbor devices within their own transmission ranges. Note that those devices that previously received the same message do not then relay the message. The above procedure is consecutively repeated until there are no more devices that cannot receive the messages within the coverage of all the relays. In summary, in the epidemic routing protocol, all devices must forward the message in the transmission range of every device, while only those devices in the range between $r_{k}$ and the maximum transmission range are chosen to forward the message in the proposed BiPAD algorithm.

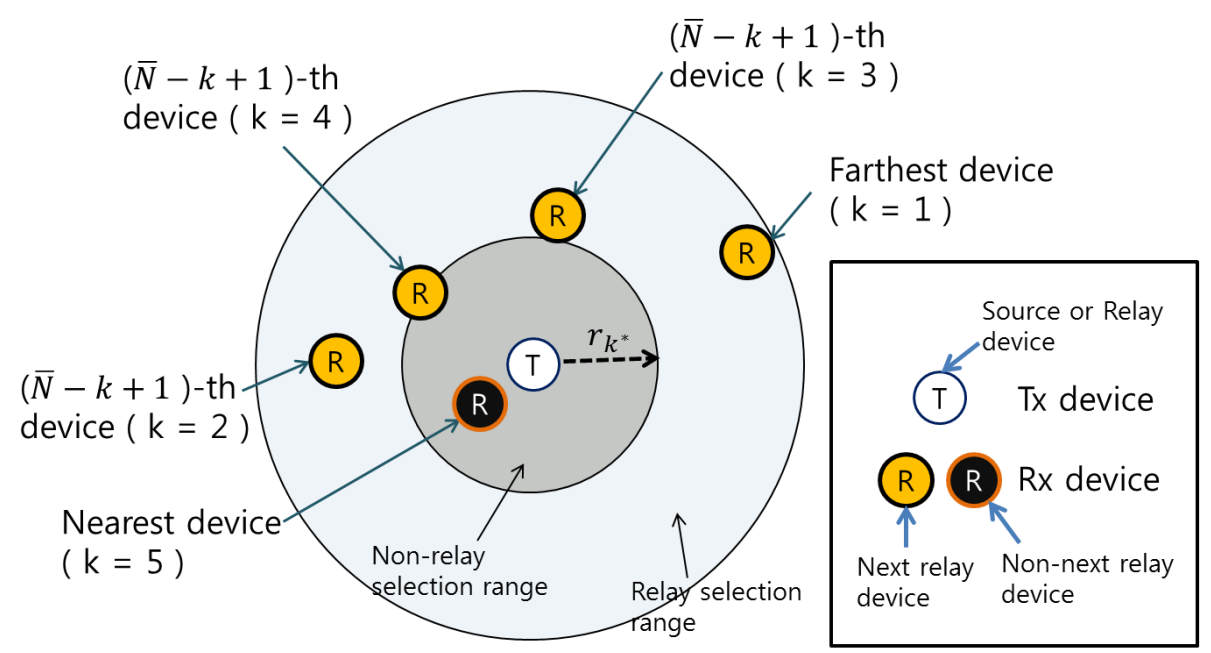

Figure 1. Relay selection with the $n$-th nearest distance when $k=4$ and $\bar{N}=5$.

\section{Simulation Results}

The use of intensive simulations allowed us to evaluate the performance of the proposed BiPAD algorithm for D2D data dissemination in comparison with that of the epidemic routing protocol. Here, the D2D source device is located at the center of the network; the other devices are uniformly distributed; and $R$ is set to $1000 \mathrm{~m}$. In the proposed algorithm, the D2D source generates and sends the message to neighboring devices within its transmission range, while the receivers within the relay selection range of BiPAD only disseminate the received message. 
Figure 2 shows the message delivery ratio against $\bar{R}$ when $N=100$. The message delivery ratio is the ratio of the number of devices that received the message at least once to the total number of devices. It is shown that the message delivery ratio increases with the value of $k$. It is notable that when $k \geq 10$, the message delivery ratio of the proposed BiPAD algorithm is generally close to that of the epidemic routing protocol. Thus, the overlapping of the graphs occurs. Figure 3 also shows the total number of received messages against $\bar{R}$ when $N=100$. Similar to Figure 2, the total number of received messages increases with $k$ because relatively more relays forward messages to their neighboring devices. However, when $k \leq 10$, we note that the total number of forwarded messages under the proposed algorithm is clearly lower than that of the epidemic routing protocol. This means that the proposed BiPAD algorithm outperforms the epidemic routing protocol with respect to the forwarding efficiency. Figure 4 demonstrates the forwarding efficiency of the proposed and conventional algorithms when $N=100$. The forwarding efficiency is defined as the ratio of the total number of received messages to the number of received devices. Here, the number of received devices relates to devices receiving messages from the source device and the relays at least once. For example, when $\bar{R}=500$, the forwarding efficiency of the proposed BiPAD algorithm shows a remarkable increase because the number of relays reduces from 3-4 at this point. This indicates that the proposed algorithm can be used to reduce the number of relays required.

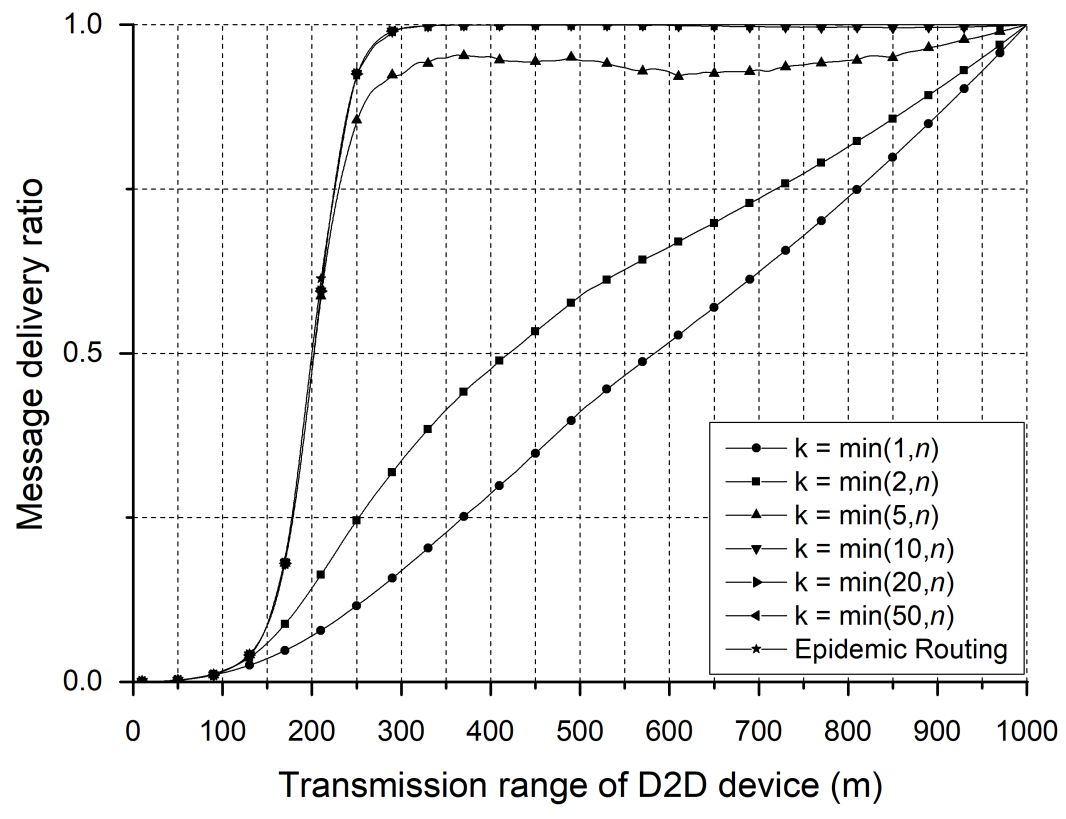

Figure 2. Message delivery ratio vs. $\bar{R}$ when $N=100$.

Figure 5 compares the performance between the proposed BiPAD and the heuristic algorithm where the heuristic algorithm exactly chooses $k$ relays that are located at the boundary of the transmission range. However, if there is no receivable device within the transmission range of the chosen relay, it no longer forwards the message to its neighboring devices. Figure $5 \mathrm{a}-\mathrm{c}$ shows the message delivery ratio, the total number of received messages and forwarding efficiency versus $\bar{R}$ when $N=100$. Even though the proposed BiPAD algorithm allows devices further away than the $k$-th furthest distance from the source device to forward a message to their neighbors, the number of relays actually used is mostly less than the calculated $k$-value. Thus, the message delivery ratio and the total number of received messages of the proposed algorithm could be less than those of the heuristic algorithm. On the other hand, the forwarding efficiency of the proposed algorithm is larger than that of the heuristic algorithm when the transmission range is less than $500 \mathrm{~m}$. That is, the proposed algorithm can obtain better forwarding efficiency with relatively less information to select desired relay devices. 


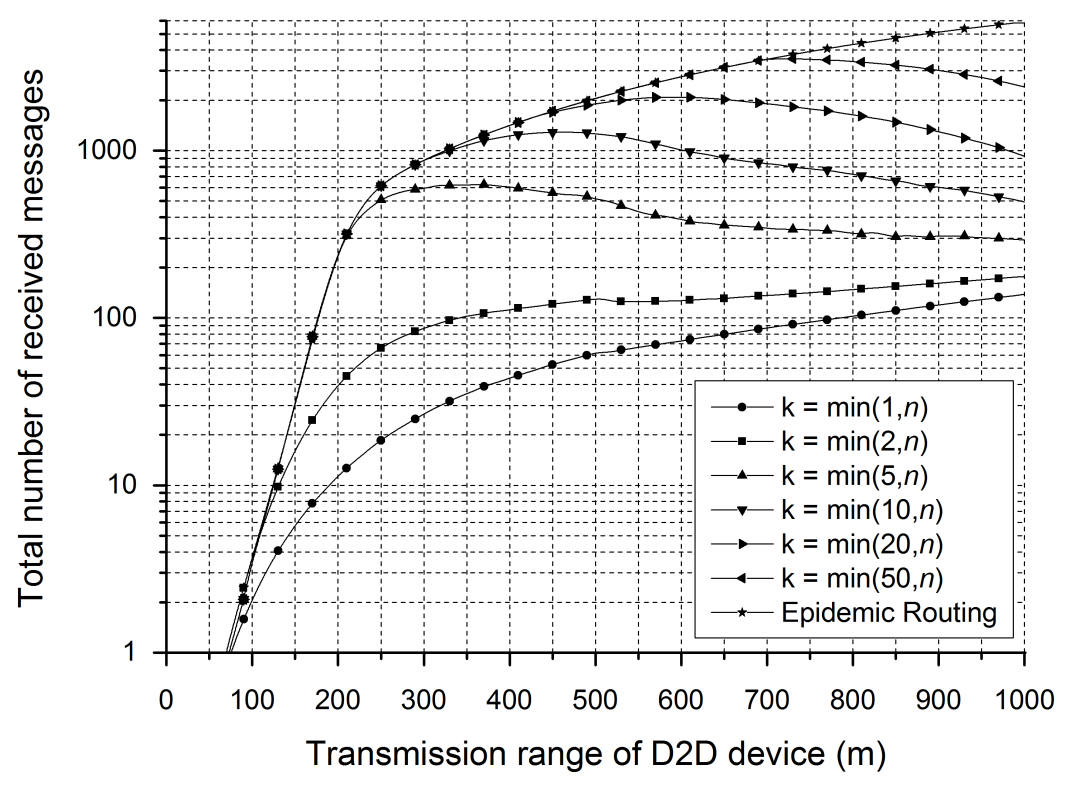

Figure 3. Total number of received messages vs. $\bar{R}$ when $N=100$.

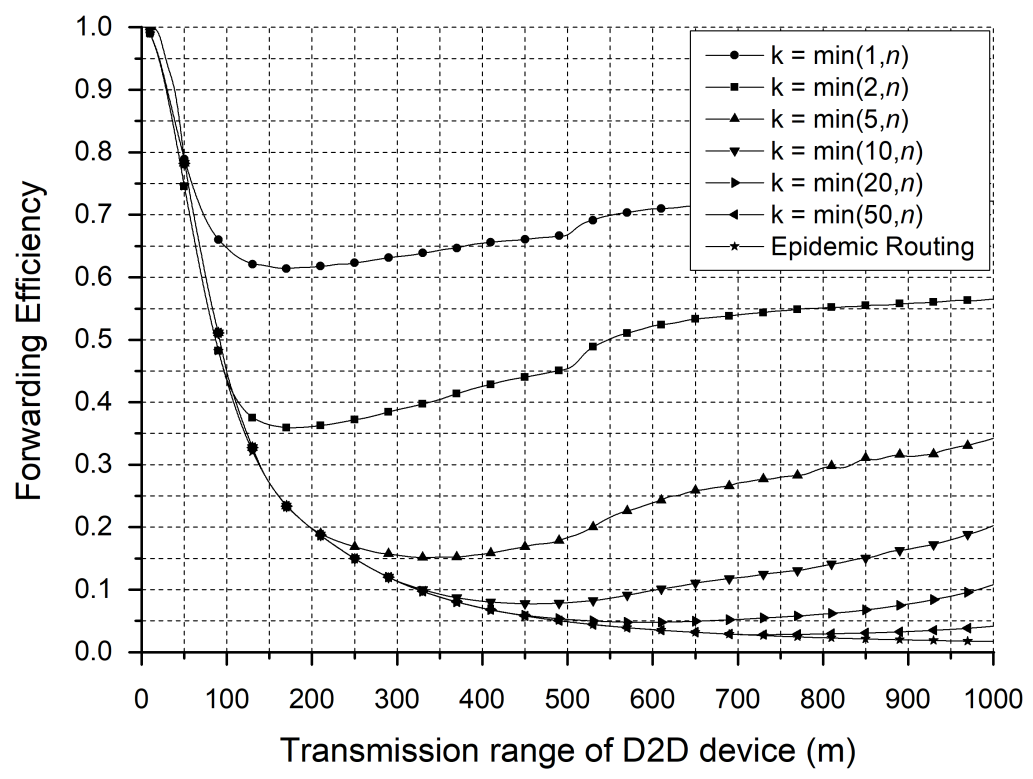

Figure 4. Forwarding efficiency vs. $\bar{R}$ when $N=100$.

Figure 6 shows the minimum value of $k$ required to achieve the same message delivery ratio as the epidemic routing protocol. This figure may provide an insight into determining the minimum number of required relays by adaptively adjusting the $k$ value to achieve almost the same message delivery ratio as the epidemic routing protocol. In Figure 6, 'Anal.'denotes the real value of $k$ used to calculate $r_{k}$, while 'Sim.' denotes the number of relays actually used in the simulations. Accordingly, the number of relays is more or less the same as the 'Anal.' $k$ value, although we note the tendency in each graph for the 'Sim.' value to follow the 'Anal.' value. When $\bar{R}$ is large, the 'Anal.' $k$-value is less than the 'Sim.' $k$-value because of the considerable overlap among the transmission ranges and the transmissions beyond the coverage of the network as a whole. 


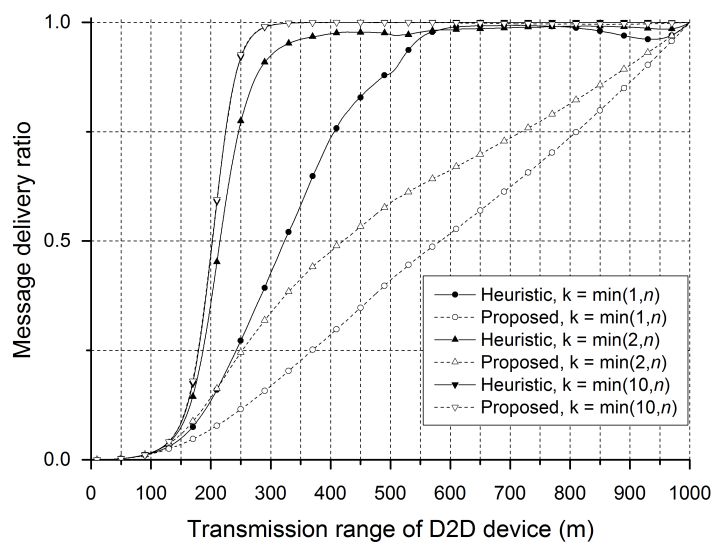

(a) Message delivery ratio

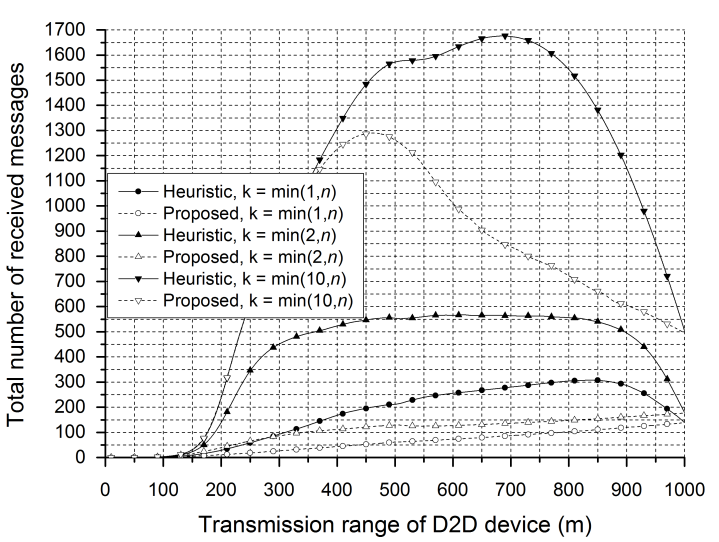

(b) Total number of received msgs

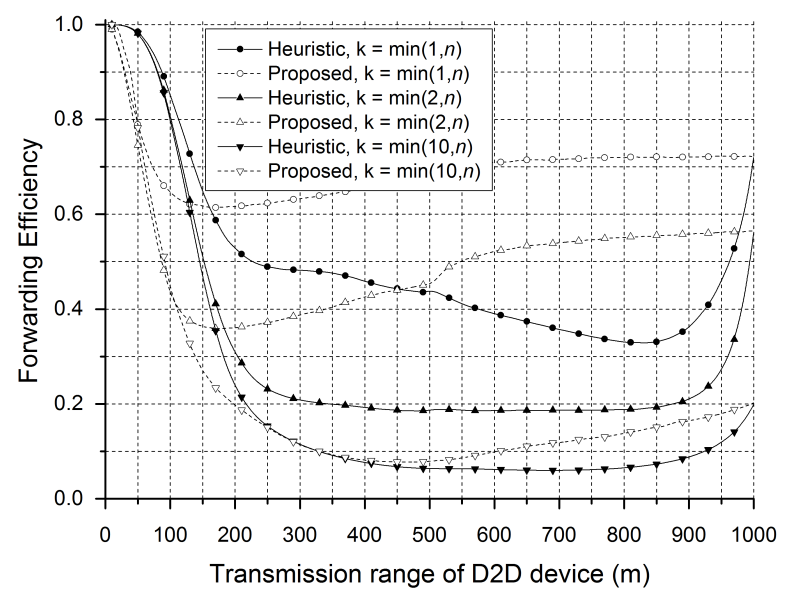

(c) Forwarding efficiency

Figure 5. Message delivery ratio, total number of received messages and forwarding efficiency vs. $\bar{R}$ when $N=100$.

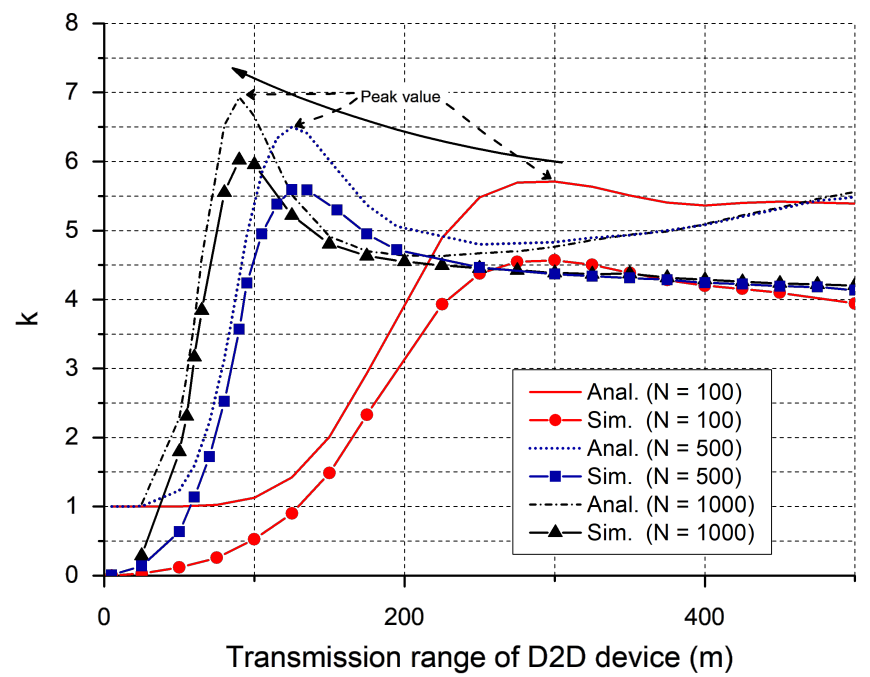

Figure 6. Minimum value of $k$ required to achieve the same message delivery ratio as the epidemic routing protocol. Sim, simulation. 


\section{Conclusions}

We have demonstrated an efficient D2D data dissemination method for reducing excessive overlap between transmission coverage while maximizing the message delivery ratio. The proposed BiPAD algorithm causes devices to forward received messages in consideration of the $k$-th furthest distance from the source device based on BPP, thereby resolving problems caused by coverage overlap and unnecessary energy consumption. We used simulations to demonstrate that the proposed BiPAD algorithm can reduce the number of received messages while ensuring the required message delivery ratio by adaptively setting the parameter $k$. Using the proposed BiPAD algorithm, the source device can adjust the number of forwarding devices by adaptively adjusting $r_{k}$ based on BPP regardless of the exact number of users within its transmission coverage. For further work, the influence spreading in social networks and the energy-aware data dissemination in the opportunistic D2D networks could be jointly considered as one of the major challenges for social-based behavior.

Author Contributions: Conceptualization, H.L. Investigation, S.H. Methodology, K.L. and H.-H.C. Supervision, H.L. Writing original draft, S.H. Writing review and editing, K.L., H.-H.C. and H.L.

Funding: This research was supported in part by the Basic Science Research Program through the National Research Foundation of Korea (NRF) funded by the Ministry of Education (NRF-2016R1D1A1B03935902) and in part by 'The Cross-Ministry Giga KOREA Project' grant funded by the Korean government (MSIT) (No. GK18S0400, Research and Development of Open 5G Reference Model.

Conflicts of Interest: The authors declare no conflict of interest.

\section{References}

1. Series, M. IMT Vision-Framework and Overall Objectives of the Future Development of IMT for 2020 and Beyond; Recommendation ITU-R: Geneva, Switzerland, 2015.

2. Andrews, J.G.; Buzzi, S.; Choi, W.; Hanly, S.V.; Lozano, A.; Soong, A.C.; Zhang, J.C. What will 5G be? IEEE J. Sel. Areas Commun. 2014, 32, 1065-1082. [CrossRef]

3. Boccardi, F.; Heath, R.W.; Lozano, A.; Marzetta, T.L.; Popovski, P. Five disruptive technology directions for 5G. IEEE Commun. Mag. 2014, 52, 74-80. [CrossRef]

4. Asadi, A.; Wang, A.Q.; Mancuso, V. A survey on device-to-device communication in cellular networks. IEEE Commun. Surv. Tutor. 2014, 16, 1801-1819. [CrossRef]

5. Liu, J.; Kato, N.; Ma, J.; Kadowaki, N. Device-to-device communication in LTE-advanced networks: A survey. IEEE Commun. Surv. Tutor. 2015, 17, 1923-1940. [CrossRef]

6. Choi, K.W.; Han, Z. Device-to-device discovery for proximitybased service in LTE-advanced system. IEEE J. Sel. Areas Commun. 2015, 33, 55-66. [CrossRef]

7. Haus, M.; Waqas, M.; Ding, A.Y.; Li, Y.; Tarkoma, S.; Ott, J. Security and privacy in device-to-device (D2D) communication: A review. IEEE Commun. Surv. Tutor. 2017, 19, 1054-1079. [CrossRef]

8. Huang, J.; Xing, C.; Qian, Y.; Haas, Z.J. Resource allocation for multicell device-to-device communications underlaying 5G networks: A game theoretic mechanism with incomplete information. IEEE Trans. Veh. Technol. 2018, 67, 2557-2570. [CrossRef]

9. Chakchouk, N. A survey on opportunistic routing in wireless communication networks. IEEE Commun. Surv. Tutor. 2015, 17, 2214-2241. [CrossRef]

10. Han, S.; Lee, H.; Kim, J.; Lee, W. On the Connectivity in Opportunistic D2D Networks with Hierarchical/Non-Hierarchical Clustering. In Proceedings of the IEEE GLOBECOM'16 Workshop on Wireless for Big Data and Big Data for Wireless, Washington, DC, USA, 4-8 December 2016.

11. Chi, K.; Huang, L.; Li, Y.; Zhu, Y.; Tian, X.; Xia, M. Efficient and reliable multicast using device-to-device communication and network coding for a $5 \mathrm{G}$ network. IEEE Netw. 2017, 31, 78-84. [CrossRef]

12. Chen, G.; Tang, J.; Coon, J.P. Optimal routing for multi-hop social based d2d communications in the internet of things. IEEE Int. Things J. 2018, 5, 1880-1889. [CrossRef]

13. Shaikh, F.S.; Wismuller, R. Routing in multi-hop cellular device-to-device (D2D) networks: A survey. IEEE Commun. Surv. Tutor. 2018. [CrossRef]

14. Aldawsari, B.; Baker, T.; England, D. Trusted Energy-Efficient Cloud-Based Services Brokerage Platform. Int. J. Intell. Comput. Res. 2015, 6, 630-639. [CrossRef] 
15. Baker, T.; Asim, M.; Tawfik, H.; Aldawsari, B.; Buyya, R. An energy-aware service composition algorithm for multiple cloud-based IoT applications. J. Netw. Comput. Appl. 2017, 89, 96-108. [CrossRef]

16. Baker, T.; Ngoko, Y.; Tolosana-Calasanz, R.; Rana, O.F.; Randles, M. Energy Efficient Cloud Computing Environment via Autonomic Meta-director Framework. In Proceedings of the 2013 Sixth International Conference on IEEE Developments in eSystems Engineering, Abu Dhabi, UAE, 16-18 December 2013; pp. 198-203.

17. Baker, T.; Al-Dawsari, B.; Tawfik, H.; Reid, D.; Ngoko, Y. GreeDi: An energy efficient routing algorithm for big data on cloud. Ad. Hoc. Netw. 2015, 35, 83-96. [CrossRef]

18. Baker, T.; Lamb, D.; Taleb-Bendiab, A.; Al-Jumeily, D. Facilitating Semantic Adaptation of Web Services at Runtime Using a Meta-Data Layer. In Proceedings of the IEEE Developments in eSystems Engineering, London, UK, 6-8 September 2010; pp. 231-236.

19. Vahdat, A.; Becker, D. Epidemic Routing for Partially Connected Ad Hoc Networks; Technical Report CS-200006; Duke University: Durham, NH, USA, 2000.

20. Erramilli, V.; Crovella, M.; Chaintreau, A.; Diot, C. Delegation forwarding. In Proceedings of the 9th ACM International Symposium on Mobile Ad Hoc Networking and Computing, Hong Kong, China, 26-30 May 2008.

21. Lindgren, A.; Doria, A.; Schelen, O. Probabilistic Routing in Intermittently Connected Networks. In Service Assurance with Partial and Intermittent Resources; Springer: Berlin/Heidelberg, Germany, 2004; Volume 3126, pp. 239-254.

22. Hui, P.; Crowcroft, J.; Yoneki, E. BUBBLE Rap: Social-based forwarding in delay-tolerant networks. IEEE Trans. Mob. Comput. 2011, 10, 1576-1589. [CrossRef]

23. Srinivasa, S.; Haenggi, M. Distance distributions in finite uniformly random networks: Theory and applications. IEEE Trans. Veh. Technol. 2010, 59, 940-949. [CrossRef]

24. Press, W.H.; Teukolsky, S.A.; Vetterling, W.T.; Flannery, B.P. Numerical Recipes 3rd Edition: The Art of Scientific Computing; Cambridge University Press: New York, NY, USA, 2007.

(C) 2018 by the authors. Licensee MDPI, Basel, Switzerland. This article is an open access article distributed under the terms and conditions of the Creative Commons Attribution (CC BY) license (http:/ / creativecommons.org/licenses/by/4.0/). 